\title{
Recording of living cell membrane depolarisation with AIGaN/GaN sensor
}

\author{
A.Podolska ${ }^{1}$, R. Seeber ${ }^{2}$, K. Pfleger ${ }^{2}$, U.K. Mishra ${ }^{3}$, G.Parish ${ }^{1}$ and B. Nener ${ }^{1}$ \\ ${ }^{1}$ School of Electrical, Electronic and Computer Engineering, The University of Western Australia, \\ Crawley, WA 6009, Australia \\ ${ }^{2}$ Laboratory for Molecular Endocrinology - GPCRs, Western Australian Institute for Medical Research \\ (WAIMR), and Centre for Medical Research, The University of Western Australia, Nedlands, WA 6009, \\ Australia \\ ${ }^{3}$ Department of Electrical and Computer Engineering, University of California, Santa Barbara, CA \\ 93106 \\ podola01@student.uwa.edu.au
}

\begin{abstract}
:
This work reports on $\mathrm{AlGaN} / \mathrm{GaN}$-based sensor development and application to biological sensing. Living cells were seeded on the sensitive region of encapsulated devices for 30 minutes before membrane depolarisation with $\mathrm{KCl}$. To confirm cell activity $\mathrm{KCl}$ was also added to devices in physiological salt solution with no cells. The resulting responses were recorded in both cases and demonstrated significant differences in profile and amplitude. Since the AIGaN/GaN structure itself is ion-sensitive, a chemical signal is expected even in the absence of cells. The depolarisation signal observed for the devices with cells is thus expected to incorporate this chemical response.
\end{abstract}

Key words: $A I G a N / G a N$, sensors, biological sensing, living cells based sensing.

\section{Introduction}

AIGaN/GaN sensor technology is a successor to silicon based field effect transistor (FET) based sensors, with higher chemical stability in liquids and better compatibility with living cells [1]. Therefore $\mathrm{AIGaN} / \mathrm{GaN}$ heterostructures are promising for the development of biochemical sensors employing extracellular signal recording. In the last decade $A I G a N / G a N$ sensors have been demonstrated for recording of cell action potential, non-invasive cell electrophysiological measurements and electrical stimulation of cell culture. However, the strength of the recorded signal was often only in the range of tens to hundreds of micro volts [2-4]. In this study we demonstrate a high amplitude (milli volts) signal during stimulated membrane depolarisation of living cells via exposure to the $\mathrm{KCl}$. The absence of this high amplitude signal in the control experiment confirmed that the signal corresponded to an extracellular event associated with the depolarisation.

\section{Experimental Methods and Results}

Ungated devices were fabricated on standard AIGaN/GaN wafers with $2 \mathrm{~nm}$ GaN capping layer. The measurements were performed on packaged four point bar structure devices with sensing areas of $1.25 \times 0.5 \mathrm{~mm}$, at constant current of $100 \mu \mathrm{A}$ using a DT82E DataTaker and constant current source. All measurements were performed inside a custom-built environmental chamber under constant temperature $\left(37^{\circ} \mathrm{C}\right)$ and constant light conditions. Standard cleaning was applied to the sensor surface (acetone, isopropyl alcohol, DI water and $\mathrm{N}_{2}$ blow-dry) after device fabrication and between measurements.

Human Coronary Artery Endothelial Cells (HCAEC) were cultured in MesoEndo Cell Growth Medium and re-suspended in standard physiological salt solution. Living cells in physiological salt solution $(500 \mathrm{uL})$ were seeded on the device sensitive area 30 minutes before depolarization, which allows cells to attach to the sensitive area of device. To achieve cell membrane depolarisation $45 \mathrm{mM}$ of $\mathrm{KCl}$ was added to the cell medium and the resulting signal was recorded (repeated three times).

For the measurement configuration used here, exposure of the surface to charge, including ionic solutions, leads to a shift of the surface potential and thus to a change in the sheet carrier density of the two dimensional electron gas (2DEG) located at the $\mathrm{AlGaN} / \mathrm{GaN}$ interface. This change is directly recorded through a time dependent measurement of potential drop across the sensitive area for constant applied current. 
To identify the nature of the observed signal a control experiment with physiological salt solution but no living cells was undertaken. The device sensitive area was exposed to $500 \mu \mathrm{L}$ of physiological salt solution and $45 \mathrm{mM}$ of $\mathrm{KCl}$ was added (repeated three times). The resulting signal demonstrated that the devices are, as expected, sensitive to changes in ionic concentration. However, the profile and amplitude of the control experiment signal is substantially different from the signal when living cells are present. Both experimental results can be seen in Fig.1. Therefore we can state that the $\mathrm{AlGaN} / \mathrm{GaN}$ sensors presented here are capable of recording biological signals, in particular living cell membrane depolarisation.

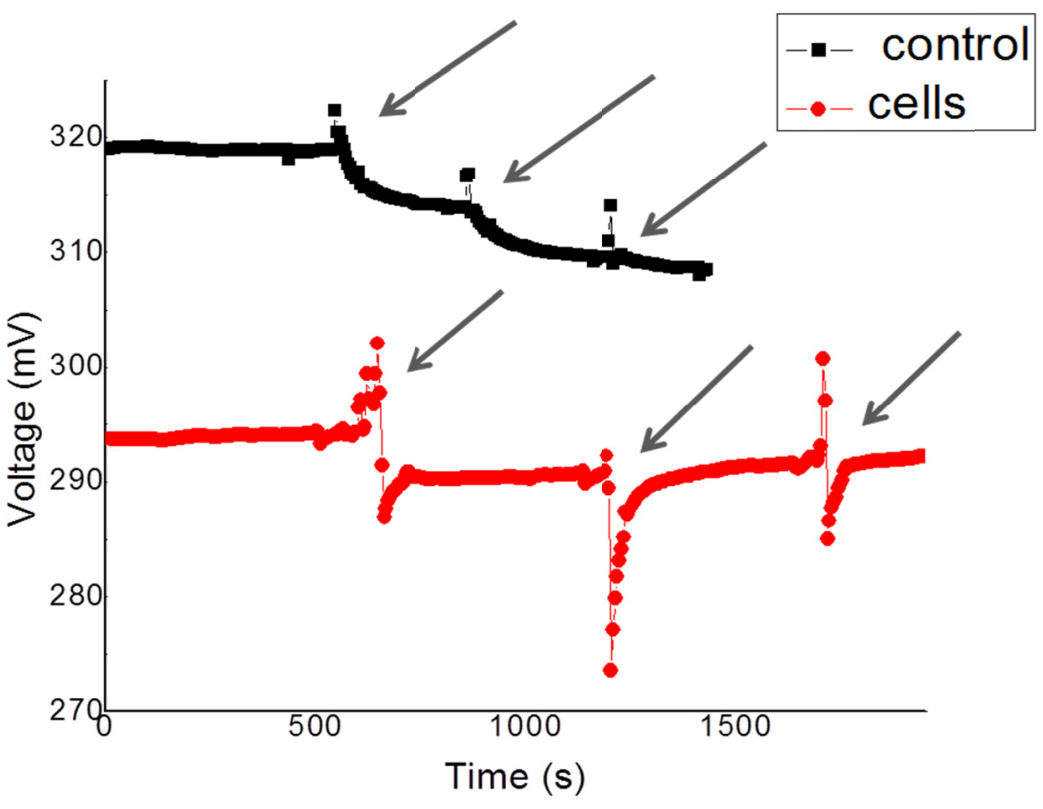

Fig. 1. Device responses to addition of $45 \mathrm{mM}$ of $\mathrm{KCl}$, for devices with cultured cells ("cells") and devices in physiological salt solution only ("control"). Data was obtained by making time dependent measurements of voltage across the sensitive area of device under constant current of $100 \mu \mathrm{A}$.

\section{Conclusion}

An AlGaN/GaN-based sensor has been demonstrated to show sensitivity to $\mathrm{KCl}$ and physiological salt solution both with and without the presence of living cells. However signals recorded with cells seeded on the sensitive area of packaged devices had higher amplitudes and different profiles and thus are considered to contain both chemical (ion concentration) and biological (cell depolarisation) components.

\section{Acknowledgements}

This work was partially funded by the Australian Research Council: DP0988241 and FT100100271, William and Marlene Schrader Postgraduate Scholarship for Biomedical Engineering with the scientific and technical assistance of the Australian Microscopy \& Microanalysis Research Facility at the Centre for Microscopy, Characterisation \& Analysis and Western Australian Node of the Australian National Fabrication Facility. Furthermore we acknowledge Angela Rizzi's group in the
Faculty of Physics at Georg-August University of Goettingen and S.P. DenBaars' group at University of California Santa Barbara for the growth of the AIGaN/GaN heterostructures.

\section{References}

[1] I. Cimalla, F. Will, K. Tonisch, M. Niebelschütz, V. Cimalla, V. Lebedev, G. Kittler, M. Himmerlich, S. Krischok, J.A. Schaefer, M. Gebinoga, A. Schober, T. Friedrich, O. Ambacher, Sensors and Actuators B: Chemical , 123, 2, 740-748 ( 2007); doi:10.1016/j.snb.2006.10..030

[2] G. Steinhoff, B. Baur, G. Wrobel, S. Ingebrandt, A. Offenhäusser, A. Dadgar, A. Krost, M. Stutzmann, M. Eickhoff, Appl. Phys. Lett. 86, 033901 (2005); doi:10.1063/1.1853531

[3] J. Yu, S. Kumar Jha, L. Xiao, Q. Liu, P. Wang, C Surya, M. Yang, Biosensors and Bioelectronics, 23, 4, 513- 519 (2007); doi:10.1016/j.bios.2007.06.014

[4] H. Witte, C. Warnke, T. Voigt, A. de Lima, I. Ivanov, T. R. Vidakovic-Koch, K Sundmacher, A. Krost, J. Phys. D: Appl. Phys., 44, 355501 (2011); doi:10.1088/0022-3727/44/35/355501 